\title{
Diffusion-induced Ramsey narrowing
}

\author{
Yanhong Xiao, ${ }^{1}$ Irina Novikova, ${ }^{1}$ David F. Phillips, ${ }^{1}$ and Ronald L. Walsworth ${ }^{1,2}$ \\ ${ }^{1}$ Harvard-Smithsonian Center for Astrophysics, Cambridge, Massachusetts, 02138 \\ ${ }^{2}$ Department of Physics, Harvard University, Cambridge, Massachusetts, 02138
}

(Dated: December 17, 2018)

\begin{abstract}
A novel form of Ramsey narrowing is identified and characterized. For long-lived coherent atomic states coupled by laser fields, the diffusion of atoms in-and-out of the laser beam induces a spectral narrowing of the atomic resonance lineshape. Illustrative experiments and an intuitive analytical model are presented for this diffusion-induced Ramsey narrowing, which occurs commonly in optically-interrogated systems.
\end{abstract}

PACS numbers: 42.50.Gy, 32.70.Jz, 42.50.Md

The lifetime of an atomic coherence is often limited by the finite interaction time between the atoms and resonant radiation: e.g., by atomic motion through a laser beam. For atoms constrained to diffuse in a buffer gas, this interaction time is usually estimated by the lowest order diffusion mode, which leads to the typical Lorentzian lineshape, but implicitly assumes that atoms diffuse out of the laser beam and do not return 1, 2, 3]. However, when other decoherence effects are small, atoms can diffuse out of the interaction region and return before decohering. That is, atoms can evolve coherently in the dark (outside of the laser beam) between periods of interaction (inside the laser beam), in analogy to Ramsey spectroscopy [4]. In many cases of interest, diffusing atoms can spend a majority of their coherence lifetime in the dark, which induces a significant spectral narrowing of the center of the atomic resonance lineshape.

In the present Letter, we identify this "diffusioninduced Ramsey narrowing" as a general phenomenon, which we characterize through demonstration experiments using Electromagnetically Induced Transparency (EIT) in warm $\mathrm{Rb}$ vapor, and with an intuitive analytical model of the repeated diffusive return of atomic coherence to the laser beam (see Fig. 1). The effects identified here are particularly important for atomic frequency standards 5] and for dynamic light-matter interactions such as slow and stored light in atomic vapor [6], but to date have only been treated in a few special cases [7, 8].

EIT results from optical pumping of atoms into a noninteracting "dark" state for two optical fields that are in two-photon Raman resonance with a pair of metastable ground states of the atomic system [9, 10]. EIT gives rise to a narrow transmission resonance for the optical fields, with a minimum spectral width set by the rate of decoherence between the two ground states constituting the "dark" state. To characterize diffusion-induced Ramsey narrowing using EIT, we employed a diode laser operating at $795 \mathrm{~nm}$ on the ${ }^{87} \mathrm{Rb} D 1$ transition to drive the atoms into EIT resonance between the $F=2$ and $F=1$ hyperfine levels of the electronic ground state. The beam passed through an enriched ${ }^{87} \mathrm{Rb}$ vapor cell (2.5 $\mathrm{cm}$ diameter, $5 \mathrm{~cm}$ length, Ne buffer gas) which was heated to approximately $45^{\circ} \mathrm{C}$ to create optically thin (a)

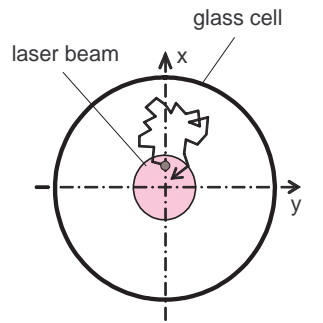

magnetic field gradient

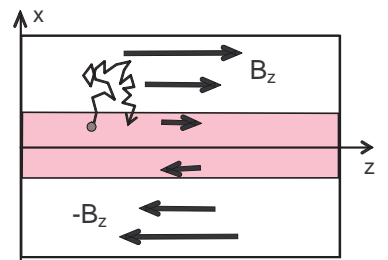

(b)

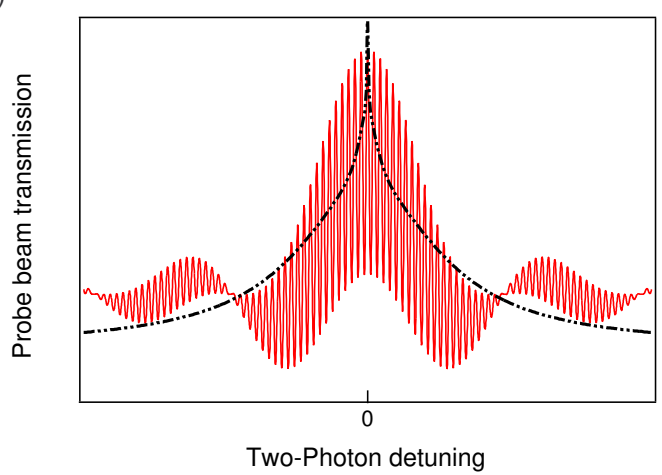

FIG. 1: (a) Left: an example path for atoms diffusing in and out of the laser beam. Right: in some of the measurements reported here, a transverse gradient in the longitudinal magnetic field was applied to the atomic vapor cell in order to decohere most atoms that diffused out of the laser beam. (b) Calculated EIT lineshapes for repeated diffusive return of atomic coherence to the laser beam. Solid red curve: an example lineshape for a particular diffusion history ("Ramsey sequence"), with the time spent in the laser beam $t_{i n}=\tau_{D}$ (the mean diffusion time to leave the beam given by the lowestorder diffusion mode), and the time spent out of the beam before returning $t_{\text {out }}=20 \tau_{D}$. Dashed black curve: weighted average over all Ramsey sequences.

Rb vapor $\left(n \sim 6 \times 10^{10} \mathrm{~cm}^{-3}\right)$. The cell was mounted within three layers of magnetic shields to screen external fields. Sets of coils were used as needed to provide a homogeneous longitudinal magnetic field $\left(B_{z}\right)$ and/or a transverse gradient in the longitudinal field $\left(\partial B_{z} / \partial x\right)$ as shown in Fig. 19. A photodetector measured the total light intensity transmitted through the vapor cell.

In a first set of experiments, we employed a VCSEL 
(vertical cavity surface emission laser) with a transverse Gaussian intensity profile, which was current-modulated at $3.4 \mathrm{GHz}$ to form the two optical fields necessary for EIT 12. We used a collimating lens and iris, and measured EIT lineshapes for various laser beam diameters in a cell with 3 Torr Ne buffer gas (Rb diffusion coefficient $\left.D \approx 50 \mathrm{~cm}^{2} \mathrm{~s}^{-1}\right)$. We set $B_{z}=0$ and $\partial B_{z} / \partial x=0$, such that EIT occurred for all relevant combinations of $m_{F}$ sublevels. As shown in Fig. 2 $\mathrm{k}$, the measured EIT resonance for a $1.5 \mathrm{~mm}$ diameter beam has a full-width-halfmaximum (FWHM) of $740 \mathrm{~Hz}$, whereas the calculated $\mathrm{FWHM} \approx 20 \mathrm{kHz}$ if one makes the common assumption that the coherence lifetime is set by the lowest order diffusion mode out of the beam (e.g., see [3]). As also shown in Fig. 20, the measured EIT lineshape for a $1.5 \mathrm{~mm}$ diameter beam is spectrally narrower near-resonance than a Lorentzian: this sharp central peak is the characteristic signature of diffusion-induced Ramsey narrowing. In contrast, the measured EIT resonance for a $10 \mathrm{~mm}$ diameter beam is well fit by a Lorentzian lineshape with FWHM $\approx 400 \mathrm{~Hz}$ (see Fig. 2b), which is in good agreement with the calculated FWHM using the lowest order diffusion mode, and is consistent with the small fraction of atoms that leave this relatively large diameter beam and return during the maximum coherence lifetime (set by buffer gas collisions and diffusion to the cell walls).

In a complementary set of experiments, we measured the EIT lineshape as a function of buffer gas pressure, thereby altering the $\mathrm{Rb}$ diffusion coefficient and changing the fraction of atomic coherence that evolves in the dark. (We used a slightly different apparatus than that described above. See 13] for details.) In Fig. 3] we compare measured EIT lineshapes for 5 Torr and 100 Torr Ne buffer gas $\left(D \approx 30\right.$ and $1.5 \mathrm{~cm}^{2} \mathrm{~s}^{-1}$, respectively), with a $0.8 \mathrm{~mm}$ laser beam diameter. Fits to the data are shown both for our analytical "repeated interaction model" (outlined below) and a Lorentzian lineshape. The repeated interaction model provides a good fit at both high and low buffer gas pressure, demonstrating its ability to account for the physics of diffusion-induced Ramsey narrowing. In contrast, the Lorentzian fit deviates significantly from the measurements even for 100 Torr Ne buffer gas - i.e., even under conditions of relatively slow $\mathrm{Rb}$ diffusion and reduced coherence evolution in the dark - which indicates the inadequacy of the traditional approach of assuming that atoms diffuse out of the laser beam and do not return.

Destroying the coherence of atoms that leave the laser beam should eliminate diffusion-induced Ramsey narrowing. As a demonstration of this behavior, we measured magnetic-field-sensitive EIT spectra (coupling the $F=2$, $m_{F}=1$ and $F=1, m_{F}=1$ levels) in the presence of both a longitudinal magnetic field $B_{z} \approx 43 \mathrm{mG}$ and a transverse gradient in the longitudinal magnetic field, $\partial B_{z} / \partial x$ (Fig. 团), using a $0.8 \mathrm{~mm}$ diameter laser beam and 5 Torr Ne buffer gas 13 . An appropriately chosen magnetic field gradient $(\approx 2 \mathrm{mG} / \mathrm{cm})$ induced modest inhomogeneous broadening of the EIT resonance $(\approx 400$
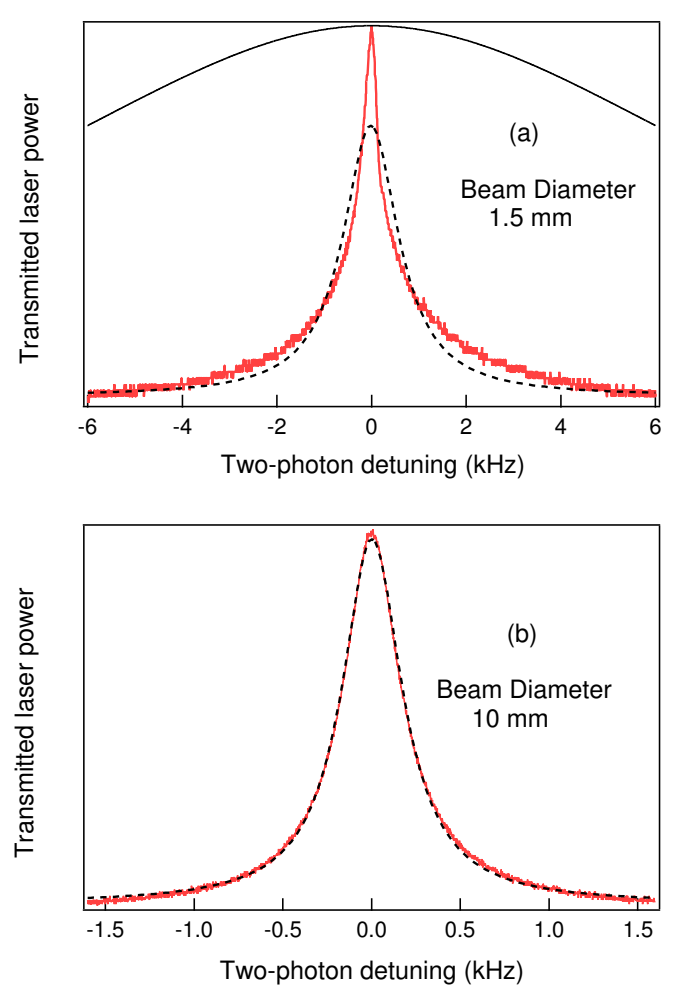

FIG. 2: Measured Rb EIT lineshapes (in red) with laser beam diameters of (a) $1.5 \mathrm{~mm}$ and (b) $10 \mathrm{~mm}$, in a 3 Torr Ne cell with $70 \mu \mathrm{W}$ laser power in the two EIT fields. Dashed lines are fits to a Lorentzian lineshape. Broad solid curve in (a) is a $20 \mathrm{kHz}$ FWHM Lorentzian, the expected lineshape for a coherence lifetime set by the lowest order diffusion mode out of the beam.

$\mathrm{Hz}$, smaller than the width of the sharp central peak) for atoms within the total volume of the laser beam [13], but caused most atoms that diffused out of the beam to lose phase coherence because of the extended period such atoms typically spend in the dark (hence probing larger differences in the magnetic field). As shown in Fig. प the characteristic sharp peak in the EIT spectrum is effectively eliminated by application of the magnetic field gradient.

We found good agreement between our measurements and numerical calculations of the Maxwell-Bloch equations, which describe the atom-light interaction, coupled to the diffusion equation, which describes the atomic motion. We also developed and successfully applied a more intuitive and analytically-soluble approach - the repeated interaction model mentioned above. In this model, the atomic resonance lineshape is calculated for an atom having a specific history ("Ramsey sequence") of alternating interactions with the laser beam and evolution in the dark. The lineshape for the atomic ensemble is then determined by a weighted average of the lineshapes from different Ramsey sequences, using the distributions of times spent in and out of the laser beam $\left(t_{\text {in }}\right.$ and $\left.t_{\text {out }}\right)$ 

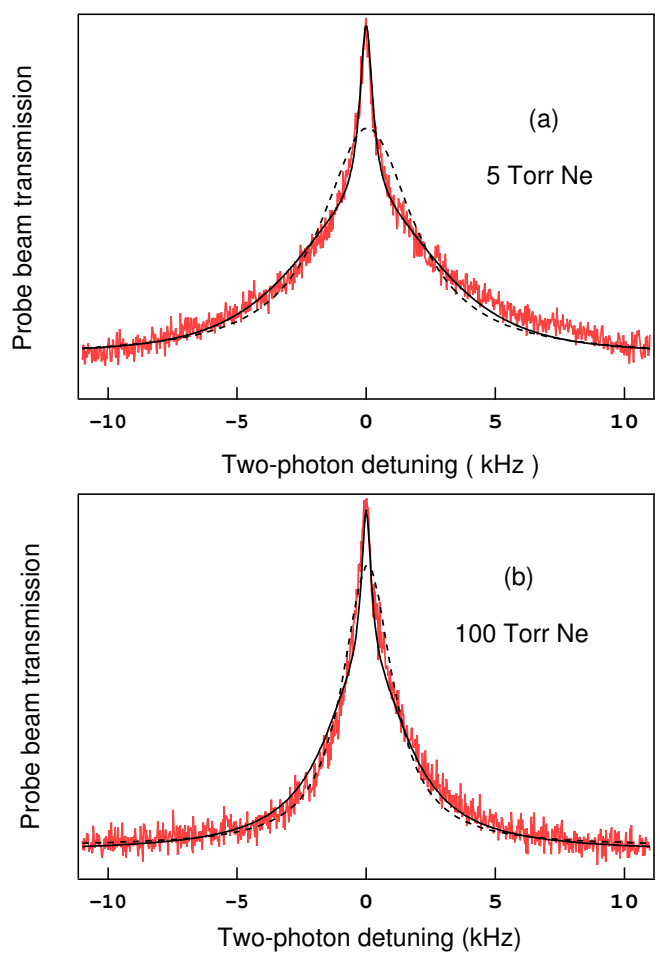

FIG. 3: Measured Rb EIT lineshapes (in red) at (a) 5 Torr and (b) 100 Torr Ne buffer gas pressure, with $22 \mu \mathrm{W}$ laser power and $0.8 \mathrm{~mm}$ beam diameter. Dashed lines are fits to a Lorentzian lineshape. Solid lines are results from the repeated interaction model.

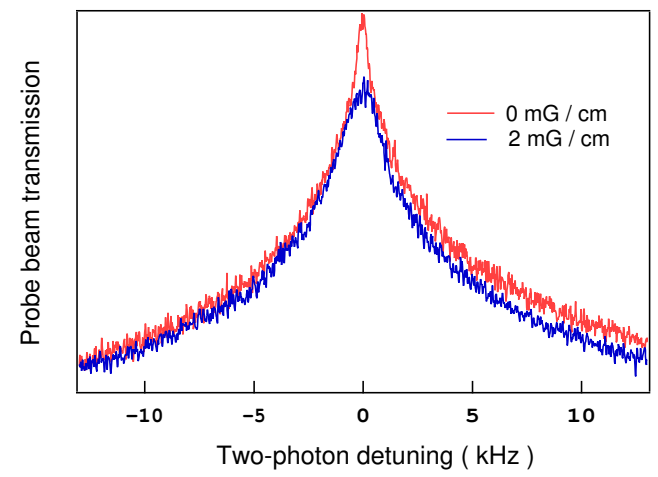

FIG. 4: Measured modification of the Rb EIT lineshape by application of a transverse magnetic field gradient $\partial B_{z} / \partial x=$ $2 \mathrm{mG} / \mathrm{cm}$ in a 5 Torr Ne buffer gas cell with $0.8 \mathrm{~mm}$ laser beam diameter. The field gradient suppresses the sharp peak caused by diffusion-induced Ramsey narrowing, but leaves the broader EIT lineshape largely unaffected.

as determined from the diffusion equation; see Fig. 5 With this approach, the atomic motion and the atomic response to laser fields are decoupled, which dramatically simplifies the calculation and allows for an analytical solution.

For example, Fig. 1p shows the EIT lineshape calculated analytically for one particular Ramsey sequence,

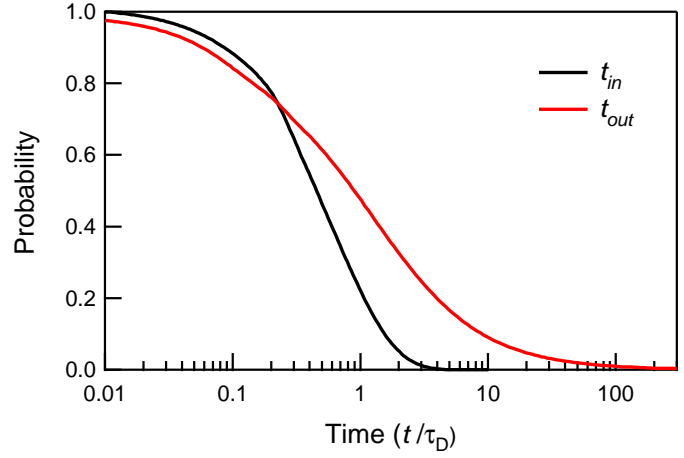

FIG. 5: Probability distributions, calculated from the diffusion equation, for an atom to spend time $t_{i n}$ inside a laser beam with a step-like transverse intensity profile, and then to spend time $t_{\text {out }}$ outside of the laser beam before returning. Times are expressed relative to $\tau_{D}$, the mean diffusion time to leave the beam given by the lowest-order diffusion mode.

as well as the ensemble lineshape determined from the weighted average over Ramsey sequences. Under the assumptions that an atom spends equal time $t_{i n}$ in the laser beam before and after diffusing in the dark for a period $t_{\text {out }}$, as well as a large difference in intensity between the two EIT optical fields, the analytical expression for the weak EIT field's transmission T as a function of twophoton Raman detuning $\Delta$ is given by:

$$
\begin{aligned}
\mathrm{T}(\Delta)= & \mathrm{T}_{0}+\frac{\kappa\left|\Omega_{d}\right|^{2} \eta}{\Delta^{2}+\Gamma^{2}}\left(-\Gamma+\sqrt{\Delta^{2}+\Gamma^{2}} \times\right. \\
& \left\{e^{-\Gamma t_{\text {in }}} \cos \left[\Delta \cdot t_{\text {in }}+\phi_{\Delta}\right]-\right. \\
& e^{-\Gamma t_{\text {in }}-\Gamma_{0} t_{\text {out }}} \cos \left[\Delta \cdot\left(t_{\text {out }}+t_{\text {in }}\right)+\phi_{\Delta}\right]+ \\
& \left.\left.e^{-2 \Gamma t_{\text {in }}-\Gamma_{\text {o }} t_{\text {out }}} \cos \left[\Delta \cdot\left(2 t_{\text {in }}+t_{\text {out }}\right)+\phi_{\Delta}\right]\right\}\right) .
\end{aligned}
$$

Here $T_{0}$ is the background transmission far from twophoton Raman resonance through the optically thin cell; $\kappa=\frac{3 \pi}{16} n \lambda^{2} L / \gamma^{2}$, where $n$ is the atomic density, $\lambda$ is the optical wavelength, $L$ is the cell length, and $\gamma$ is the relaxation rate of the excited state; $\Omega_{d}$ is the Rabi frequency for the strong optical field; $\eta$ is the radiative decay rate of the excited state; $\Gamma=\Gamma_{0}+\left|\Omega_{d}\right|^{2} / 2 \gamma$ is the powerbroadened EIT linewidth in the absence of diffusioninduced Ramsey narrowing, where $\Gamma_{0}$ is the intrinsic relaxation rate of the ground-state coherence (set by buffer gas collisions, etc.); and $\tan \phi_{\Delta}=\Delta / \Gamma$. The above expression also assumes $\gamma \gg \Delta, \Gamma_{0}, \Gamma$ and $\gamma \Gamma \gg \Delta^{2}$, which are typically satisfied for EIT in warm Rb vapor. The first term in brackets in Eq. (1) is the contribution from atoms that interact with the laser beam only once. The second and third terms account for returning atoms. More generally, the time spent inside the laser beam before and after leaving may differ. Also, atoms may return to the beam more than once; each additional diffusive return will produce two extra terms similar to the last two lines in Eq. (10).

To achieve good fits to the measured EIT lineshapes with no free fitting parameters (see Fig. 31), we found 
that it is sufficient to consider Ramsey sequences limited to only one or two evolution periods in the dark. In these calculations we assumed a step-like laser profile in the transverse direction, which is a good approximation when the effective two photon Rabi period is longer than the average time atoms spend in the laser beam, so that an atom averages over the transverse Gaussian distribution of laser intensity. Details of these calculations are described in 14.

The width of the lineshape envelope for an individual Ramsey sequence, such as that shown in Fig. 1b, scales inversely with the time the atom spends in the laser beam $\left(t_{i n} \approx \tau_{D} \propto w^{2} / D\right.$, where $D$ is the $\mathrm{Rb}$ diffusion coefficient and $w$ is the beam width); whereas the width of the Ramsey fringes is inversely proportional to the free evolution time in the dark $\left(t_{\text {out }}>\tau_{D}\right)$. The sharp central peak, indicative of diffusion-induced Ramsey narrowing, emerges intuitively in this model, since only the central fringe adds constructively for all Ramsey sequences, with different diffusion times outside of the beam. The narrow width of this central peak is limited by other effects (atomic collisions, magnetic field gradients, wall collisions, etc.) which set an upper bound on the free evolution time. Since the atoms contributing most to the sharp central peak spend the majority of their time in the dark, the width of this peak is relatively insensitive to power broadening.

In general, when the laser beam diameter is small, reshaping and narrowing of the lineshape are strong, since a large fraction of the atoms participate in the diffusioninduced Ramsey process, and the free-evolution time between interactions with the laser beam can be long. For larger laser beam diameters, the Ramsey narrowing gradually disappears since a smaller fraction of the atoms can diffuse out of the beam and return before decohering due to other effects. In particular, when the laser beam diameter approaches the cell diameter, atoms diffusing out of the beam rapidly decohere due to wall collisions. (In ongoing work we are investigating the effect of coherencepreserving wall-coatings.)

We note that non-Lorentzian EIT lineshapes can appear in other circumstances. For instance, a well-known form of linewidth narrowing occurs in optically thick media due to frequency-selective absorption [15]. Alternatively, for an optically thin medium with inhomogeneous laser intensity, atoms can reach equilibrium locally in the limit of high buffer gas pressure, producing a spatial variation of the power broadening and an inhomogeneously broadened (and non-Lorentzian) lineshape [16, 17]; whereas in the limit of low buffer gas pressure, atoms can be pumped at one intensity and probed at another, leading to a non-Lorentzian lineshape dependent on the effusive time-of-flight of atoms across the sample cell [18]. These effects are qualitatively and quantitatively distinct from diffusion-induced Ramsey narrowing.

In conclusion, we identified a novel form of spectral narrowing arising from the diffusion of atomic coherence in-and-out of an optical interaction region, such as a laser beam. We characterized this "diffusion-induced Ramsey narrowing" with measurements on Electromagnetically Induced Transparency (EIT) in warm Rb vapor, and found good agreement with an intuitive analytical model based on a weighted average of distinct atomic histories in the light and the dark. This "repeated interaction model" and the spectral narrowing effects studied here are relevant to spectroscopy, quantum optics and other applications based on long-lived atomic coherences.

We are grateful to F. Canè, J. Vanier and A. B. Matsko for useful discussions, and to C. Smallwood and C. Wang for assistance in experiments and modelling. This work was supported by ONR, DARPA, and the Smithsonian Institution.
[1] W. Happer, Rev. Mod. Phys. 44, 169 (1972).

[2] E. Arimondo, Phys. Rev. A 54, 2216 (1996).

[3] M. Erhard and H. Helm, Phys. Rev. A 63, 043813 (2001).

[4] N. F. Ramsey, Molecular beams (Clarendon, Oxford, 1956).

[5] J. Vanier, to appear in Appl. Phys. B (2005).

[6] M. D. Lukin, Rev. Mod. Phys. 75, 457 (2003).

[7] A. S. Zibrov, I. Novikova and A. B. Matsko, Opt. Lett. 26, 1311 (2001).

[8] A. S. Zibrov and A. B. Matsko, Phys. Rev. A 65, 013814 (2001).

[9] E. Arimondo, in Progress in Optics edited by E. Wolf, (Elsevier Science, Amsterdam, 1996), Vol. XXXV, Chap. V, pp. 259-354.

[10] M. O. Scully, and M. S. Zubairy, Quantum Optics (Cambridge University Press, Cambridge, UK, 1997).

[11] T. Zanon, S. Guerandel, E. de Clercq, D. Holleville, N. Dimarcq, and A. Clairon, Phys. Rev. Lett. 94, 193002 (2005).
[12] C. Affolderbach, A. Nagel, S. Knappe, C. Jung, D. Wiedenmann, and R. Wynands, Appl. Phys. B 70, 407 (2000).

[13] I. Novikova, Y. Xiao, D. F. Phillips, and R. L. Walsworth, quant-ph/0505186 to appear in J. Mod. Opt. (2005).

[14] Y. Xiao, I. Novikova, D. F. Phillips, and R. L. Walsworth, to be published.

[15] M. D. Lukin, M. Fleischhauer, A. S. Zibrov, H. G. Robinson, V. L. Velichansky, L. Hollberg and M. O. Scully, Phys. Rev. Lett. 79, 2959 (1997).

[16] F. Levi, A. Godone, J. Vanier, S. Micalizio, and G. Modugno, Eur. Phys. J. D 12, 53 (2000).

[17] A. V. Taichenachev, A. M. Tumaikin, V. I. Yudin, M. Stahler, R. Wynands, J. Kitching, and L. Hollberg, Phys. Rev. A 69, 024501 (2004).

[18] E. Pfleghaar, J. Wurster, S. I. Kanorsky and A. Weis, Optics Commun. 99, 303 (1993). 